ALÉTHEIA

Enero - Diciembre. 2019, Vol. N7, pp. 31-35

DOI: 10.33539/aletheia.2019.n7.2150

\section{UNA CONDICIÓN DE VIDA, PERSONAS CON TRASTORNO DEL ESPECTRO AUTISTA}

\author{
A CONDITION OF LIFE, PEOPLE WITH \\ DISORDER OF THE AUTISTIC SPECTRUM
}

\begin{abstract}
RESUMEN
La diversidad nos enriquece, rúbrica que concierne apropiarse sobre todo en el sistema educativo peruano, en todos los niveles y ámbitos educativos, donde se encuentra diariamente diversidad de estudiantes, entre ellos a los estudiantes con trastorno del espectro autista, quienes muchos de ellos desarrollan altas capacidades, o a veces pasan desapercibidos dentro de un contexto o son contemplados con indiferencia por sus expresiones involuntarias y/o movimientos estereotipados. ¿Entonces qué labor demanda cumplir a un educador? Esta situación conlleva conocer las características presentes en cada uno y efectuar la detección temprana del TEA, a partir de la identificación brindar una atención educativa centrada en la persona, valorando sus potencialidades y habilidades, con el apoyo del equipo interdisciplinario con profesionales especializados en el tema que aborda al estudiante y su familia, a fin de brindar una respuesta adecuada a sus necesidades y logren incluirse en las aulas de diferentes instituciones educativas y a futuro conlleve a una inclusión trascendental dentro de la sociedad. Frente a ello, surge la necesidad y compromiso de educadores de brindar información básica sobre las personas con trastorno del espectro autista. Es así que en el presente artículo se contribuirá conocer más sobre el tema y sobre las características relacionadas al desarrollo de las habilidades comunicativas, flexibilidad mental y habilidades sociales.
\end{abstract}

Palabras clave: Trastorno del espectro autista (TEA), Diversidad, Aulas inclusivas.

\section{ABSTRACT}

Diversity enriches us, a heading that concerns itself, above all, in the Peruvian educational system, at all levels and educational settings, where students are daily diverse, including students with autism spectrum disorder, who many develop high capabilities, or sometimes go unnoticed within a context or are contemplated with indifference for their involuntary expressions and / or stereotyped movements. So what work demands to fulfill an educator? This situation entails knowing the characteristics present in each one and carrying out the early detection of ASD, based on the identification of providing a person-centered educational service, assessing their potential and skills, with the support of the interdisciplinary team with professionals specialized in the subject which addresses the student and his family, in order to provide an adequate response to their needs and achieve inclusion in the classrooms of different educational institutions and in the future leads to a transcendental inclusion within society. Against this, the need and commitment of educators to provide basic information on people with autism spectrum disorder arises. Thus, this article will contribute to know more about the topic and about the characteristics related to the development of communication skills, mental flexibility and social skills.

Keywords: Autism Spectrum Disorder (ASD). Diversity. Inclusive classrooms
Mg. Dora Quiroz Medina

doraquime2013@gmail.com

Universidad Femenina del Sagrado Corazón

\section{INTRODUCCIÓN}

En los últimos tiempos la sociedad vive muy de prisa, dentro de ello se ha observado en cierta oportunidad estudiantes diversos y algunos con capacidades que destacan en el grupo o a veces son automáticos en su aprendizaje, otros parecen que fueran personas sordas, pocos sociales, situaciones confusas frente al otro, ellas son las personas con Trastorno del Espectro Autista, y en varias oportunidades estar frente a ellas y ellos es un reto, pues se desconoce el cómo relacionarse, cómo comunicarse y pasan inadvertidos, pero es cuando más apoyo requieren, ya que a veces pueden estar en peligro. Además de no ser totalmente receptivos a la estimulación ambiental, las personas autistas realizan algunas estereotipias motoras, parecidas a las que muestran los niños con déficit sensorial.

En respuesta a la situación ya se cuenta en el Perú, con la Ley N ${ }^{\circ} 30150$ de Protección de personas con Trastorno del Espectro Autista (TEA) y su Reglamento el Decreto Supremo 001-2015 MIMP, desarrolla todo un marco legal que busca garantizar una intervención oportuna y de calidad a la persona con TEA, por lo que se requiere se fomente la detección y diagnóstico precoz, la intervención temprana, la protección de la salud, la educación integral, la capacitación profesional y la inserción laboral y social de las personas con TEA, al amparo de lo dispuesto por el artículo 7 de la Constitución Política del Perú y de la Ley 29973, Ley General de la Persona con 
Discapacidad. Así también, se hace referencia a los enfoques transversales del CNEB que parte por reconocer a los estudiantes de la diversidad, entre ellos personas con trastorno del espectro autista, como sujetos de derechos y no como objetos de cuidado, es decir, como personas con capacidad de defender y exigir sus derechos legalmente reconocidos. Del mismo modo, reconocer que son ciudadanos con deberes que participan del mundo social propiciando la vida en democracia. Este enfoque promueve la consolidación de la democracia que vive el país, contribuyendo a la promoción de las libertades individuales; a fortalecer la convivencia y transparencia en las instituciones educativas; a reducir las situaciones de inequidad y procurar la resolución pacífica de los conflictos.

Finalmente, ostentamos el enfoque Inclusivo o de atención a la diversidad, que centra su atención al respeto por las diferencias y disposición de enseñar y a depositar expectativas en una persona, creyendo sinceramente en su capacidad de superación y crecimiento por sobre cualquier circunstancia.

\section{1. ¿Qué es el trastorno del espectro autista?}

Es un trastorno que comienza en la niñez y dura toda la vida, de allí la determinación de ser personas con "una condición de vida". No se conocen todavía cuales son las causas etiológicas concretas del autismo, pero se continúan realizando múltiples investigaciones en este ámbito. Existen herramientas para explorar sus criterios diagnósticos, y así poder detectarlo cuanto antes, realizar la intervención temprana en las diferentes áreas de desarrollo y desenvolverse al máximo para que la persona pueda adaptarse a su ambiente.

La Asociación Americana de Psiquiatría, en el año 2013, presenta el Manual de Salud mental el DSM-V, donde tanto los términos "trastornos autistas", "trastorno de Asperger" y "trastorno desintegrativo de la infancia" han sido sustituidos por "trastorno del espectro autista", y se consideran las siguientes características fundamentales del trastorno:

- Un limitado desarrollo de la interacción social

- La comunicación claramente deficitaria,

- Y un repertorio muy restringido de actividades e intereses.

Valdez (2016), explica que este trastorno es tan variado, como la existencia de cada una de las personas; por lo tanto, el reto es aprender a detectar e intervenir oportunamente desde el rol docente, dentro y fuera del aula.

Menciona textualmente los siguientes criterios para su diagnóstico:

1. Déficits en reciprocidad socio-emocional, tales como:

- Fracaso en la conversación normal en ambos sentidos,

- Disminución en intereses, emociones o afectos compartidos.

- Fracaso en iniciar o responder a interacciones sociales.

Déficits persistentes en comunicación y en la interacción social en diversos contextos.

2. Déficits en conductas comunicativas no verbales usadas en la interacción social, tales como:

- Comunicación verbal y no verbal poco integrada,

- Poco o nulo contacto visual y del lenguaje corporal,

- Deficiencias en la comprensión y el uso de gestos,

- Falta de expresión facial y de comunicación no verbal. 
3. Déficits para desarrollar, mantener y comprender relaciones sociales, tales como:

- Dificultad en ajustar el comportamiento a diversos contextos sociales,

- Dificultades para compartir el juego imaginativo o para hacer amigos,

- Ausencia de interés por las otras personas.

1. Movimientos, uso de objetos o habla estereotipada o repetitiva, tales como:

- Estereotipias motrices simples,

- Alineación de juguetes,

- Cambio de lugar de los objetos,

- Ecolalia y Frases idiosincráticas

2. Insistencia en la monotonía, excesiva inflexibilidad a rutinas, o patrones ritualizados de comportamiento verbal y no verbal, tales como:

- Elevada angustia ante pequeños cambios,

- Dificultades con las transiciones,

- Patrones de pensamiento rígidos,

- Rituales de saludo,

- Necesidad de seguir siempre la misma ruta o de comer los mismos alimentos cada día.

intereses 0 activida-

des, que se manifies-

tan al menos dos 0

más síntomas.

3. Intereses muy restrictivos y fijos que son anormales en cuanto a su intensidad y focos de interés se refiere; por ejemplo:

- Fuerte vínculo o elevada preocupación hacia objetos inusuales,

- Intereses excesivamente circunscritos y perseverantes.

4. Híper o hiporreactividad a los estímulos sensoriales o interés inusual por los aspectos sensoriales del entorno; por ejemplo:

- Aparente indiferencia al dolor/temperatura,

- Respuesta adversa a sonidos y texturas específicas,

- Oler o tocar excesivamente objetos,

- Fascinación visual con luces o movimientos.

Los síntomas tienen que manifestarse en el periodo de desarrollo temprano.

En los diferentes contextos de alguna celebración por y para las personas con TEA, encontramos a grupos de familias que resplandecen con el uso del color azul en sus vestimentas o recursos materiales, entre ellas encontramos a ASPAU (2007), quienes mencionan que el azul a veces es "brillante como el mar en un día de verano, y otras veces, ese azul oscurece y se disipa como un mar de tempestad", de esta manera nosotros podemos reconocer que el trastorno del espectro autista es una condición cuya intensidad se manifiesta en cada persona de forma particular, es por eso que algunos autores la denominan "Autismo", porque no hay casos de autismo uno igual al otro. 
Solemos ver en las campañas de difusión del Trastorno del espectro autista (TEA) el uso del color azul, color que se ha transformado en un símbolo del autismo y que representa de alguna manera lo que viven a diario las familias y las personas con este trastorno.

\section{2. ¿Qué niveles de severidad presente el TEA se- gún el DSM 5?}

En el manual también especifica el nivel de severidad que pueden presentar las personas que presentan este trastorno, se resume en:

\section{CARACTERÍSTICAS COMUNES DEL NIÑO CON TEA}

\section{Nivel 3 requiere un apoyo muy notable.}

Déficits severos en habilidades de comunicación social verbal y no verbal que causan alteraciones severas en el funcionamiento, inicia muy pocas interacciones y responde mínimamente a los intentos de relación de otros. Por ejemplo, una persona con muy pocas palabras inteligibles que raramente inicia interacciones sociales, y que cuando lo hace, realiza aproximaciones inusuales únicamente para satisfacer sus necesidades y sólo responde a acercamientos sociales muy directos

\section{Nivel 2 Requiere u apoyo}

Déficits marcados en habilidades de comunicación social verbal y no verbal; los déficits sociales son aparentes incluso con apoyos; inician un número limitado de interacciones sociales; y responden de manera atípica o reducida a los intentos de relación de otros. Por ejemplo, una persona que habla con frases sencillas, cuya capacidad para interactuar se limita a intereses restringidos y que manifiesta comportamientos atípicos a nivel no verbal.

\section{Nivel 1 Requiere ayuda}

Las dificultades de comunicación social causan alteraciones evidentes. Muestra dificultades iniciando interacciones sociales y ofrece ejemplos claros de respuestas atípicas o fallidas a las aperturas sociales de otros. Puede parecer que su interés por interactuar socialmente está disminuido. Por ejemplo, una persona que es capaz de hablar usando frases completas e implicarse en la comunicación pero que a veces falla en el flujo de ida y vuelta de las conversaciones y cuyos intentos por hacer amigos son atípicos y generalmente fracasan.

\section{NECESIDAD EDUCATIVA ESPECIAL}

La inflexibilidad del comportamiento, la extrema dificultad afrontando cambios u otros comportamientos restringidos/repetitivos, interfieren marcadamente en el funcionamiento en todas las esferas. Gran malestar o dificultad al cambiar el foco de interés o la conducta.

El comportamiento inflexible, las dificultades para afrontar el cambio, u otras conductas restringidas/repetitivas, aparecen con la frecuencia suficiente como para ser obvios a un observador no entrenado e interfieren con el funcionamiento en una variedad de contextos. Gran malestar o dificultad al cambiar el foco de interés o la conducta.

La inflexibilidad del comportamiento causa una interferencia significativa en el funcionamiento en uno o más contextos. Los problemas de organización y planificación obstaculizan la independencia. 


\section{CONCLUSIÓN}

Las personas con autismo tienen un gran potencial. La mayoría tiene extraordinarias habilidades visuales, artísticas o académicas. Gracias al uso de tecnologías de apoyo, las personas con autismo no verbal pueden comunicarse y compartir sus capacidades no aparentes. Reconocer el talento de las personas en el espectro autista, más que centrarse en sus debilidades, es esencial para crear una sociedad que sea verdaderamente inclusiva y que el aprendizaje se dé centrado en el estudiante.

\section{REFERENCIAS}

Ley de protección de las personas con trastorno del espectro autista (TEA). Recuperado en: http://www.conadisperu.gob.pe/web/documentos/NORMAS/Ley\%2030150.pdf

Fernández Morales, Antonio Daniel. El mundo del autismo. Revista internacional de audición y lenguaje, logopedia, apoyo a la integración y multiculturalidad. ISSN: 2387-0907, Dep. Legal: J -67- 2016 Volumen 2, Número 2, abril 2016. Recuperado en: https://dialnet. unirioja.es/descarga/articulo/6940996.pdf
Minedu, Curso virtual autoformativo, Transtorno del espectro autista - módulo I. Recuperado en: http://aulavirtual.perueduca.pe/pluginfile. $\mathrm{php} / 2064474 / \mathrm{mod}$ resource/content/1/MODULO\%201.pdf

Plan Nacional para las personas con trastorno del Espectro Autista 2019 - 2021. Recuperado en: http://www.conadisperu.gob.pe/wp-content/uploads/2019/01/PLAN_TEA_20192021.pdf
Fecha de Recepción: 14 de Agosto de 2019

Fecha de Aceptación: 17 de Noviembre de 2019 